ఠ

\title{
New developments in the management of schizophrenia and bipolar disorder: potential use of cariprazine
}

This article was published in the following Dove Press journal:

Therapeutics and Clinical Risk Management

2 November 2015

Number of times this article has been viewed

Felix-Martin Werner ${ }^{1,2}$

Rafael Coveñas ${ }^{2}$

'Euro Akademie Pößneck, Higher Vocational School for Elderly Care and Occupational Therapy, Pößneck, Germany; ${ }^{2}$ Institute of Neurosciences of Castilla y León (INCYL), Laboratory of Neuroanatomy of the Peptidergic Systems (Lab. 14), University of Salamanca, Salamanca, Spain
Correspondence: Felix-Martin Werner Institute of Neurosciences of Castilla y León (INCYL), Laboratory of Neuroanatomy of the Peptidergic Systems (Lab. 14), University of Salamanca, c/Pintor Fernando Gallego, I, 37007 Salamanca, Spain

Tel +34923294400

Fax +34923294549

Email felixm-werner@versanet.de

\begin{abstract}
Cariprazine is a recently developed antipsychotic drug with a partial agonism for the $\mathrm{D}_{2}$ and $\mathrm{D}_{3}$ receptors. It shows a tenfold greater affinity for the $\mathrm{D}_{3}$ receptor. In clinical trials, its therapeutic effect has been tested in patients with an acute exacerbation of schizophrenia and in patients with acute mania in bipolar disorder. Like risperidone, cariprazine improves positive and negative schizophrenic symptoms, and ameliorates cognitive functions. Cariprazine induces extrapyramidal symptoms less often than risperidone and can cause acute akathisia. It is a prolactin-sparing antipsychotic drug and has a favorable metabolic profile. In acute mania in bipolar disorder, it treats manic symptoms significantly better than placebo. As a consequence of its improved adverse effects, cariprazine improves patients' quality of life to a greater extent than other second-generation antipsychotic drugs. Cariprazine is a promising antipsychotic drug in the treatment of schizophrenia, acute mania in bipolar disorder, and in schizophrenia with mania. In these patients, its long-term therapeutic effect and its action in comparison with other second-generation antipsychotic drugs, above all aripiprazole, remain to be tested in clinical trials.
\end{abstract}

Keywords: cariprazine, second-generation antipsychotic drug, schizophrenia, acute mania, $\mathrm{D}_{2}$ receptor, $\mathrm{D}_{3}$ receptor, partial agonism, akathisia, metabolic parameters, cognitive function

\section{Introduction to developments in the management of schizophrenia and bipolar disorder}

Psychotic disorders can be divided into schizophrenia and affective disorders. Schizophrenia is a chronic disabling disorder, which manifests as an acute psychosis with positive symptoms such as hallucinations, paranoia, and illusions. Second-generation antipsychotic drugs are the preferred drugs in the treatment of schizophrenia because they improve positive and negative schizophrenic symptoms, however, in most cases after the remission of the acute psychotic symptoms, negative symptoms such as autism, social withdrawal, and cognitive symptoms remain. Permanent medication is necessary in order to prevent a recurrence of psychotic symptoms. ${ }^{1}$ A milestone in the antipsychotic treatment of schizophrenic patients was the introduction of typical neuroleptics, such as haloperidol, $\mathrm{a}_{2}$ antagonist. Haloperidol, above all, improves positive schizophrenic symptoms, but often causes extrapyramidal side effects, weight gain, and increased prolactin levels. ${ }^{2}$ Schizophrenia is undoubtedly an inheritable disease with genetically encoded neurotransmitter alterations. The enzymes catalyzing dopamine breakdown have a reduced activity. Moreover, GABAergic and glutaminergic neurons, which have a presynaptic inhibitory action, have a decreased function in the brain regions involved in schizophrenia. ${ }^{3}$ In the mesolimbic system, 
hippocampus, and prefrontal cortex both dopamine and serotonin hyperactivity can be found in schizophrenia, whereas a stimulation of $\mathrm{D}_{2}$ and serotonin $(5-\mathrm{HT})_{2 \mathrm{~A}}$ receptors enhances psychotic symptoms. ${ }^{4-6}$ Among the secondgeneration antipsychotic drugs, combined $\mathrm{D}_{2}$ and $5-\mathrm{HT}_{2 \mathrm{~A}}$ antagonists, the most commonly used antipsychotic drugs are risperidone, olanzapine, and quetiapine. ${ }^{4}$ The affective disorder with manifestations of depressive and manic symptoms is bipolar disorder. ${ }^{1}$ In depressive patients, hypoactivity of monoamines in the brainstem and hippocampus occurs. Antidepressant drugs, which block the reuptake of serotonin and/or noradrenaline, fail to improve the lack of energy, interest, and pleasure. Only antidepressant drugs, which block the reuptake of noradrenaline and dopamine or triple reuptake inhibitors, can improve the decreased effects, ie, the loss of energy, pleasure, and interest. ${ }^{7}$ In manic patients, hippocampal dopaminergic neurons show alternating hypo- and hyperactivity with transient normal activity via $\mathrm{D}_{2}$ receptors that can be improved by administering cariprazine. ${ }^{8}$ The alterations in monoamines are due to polymorphisms of the monoamine transporter genes. ${ }^{9}$ Depressive symptoms are treated by monoamine reuptake inhibitors, for example, selective serotonin reuptake inhibitors or serotonin and noradrenaline reuptake inhibitors. Patients with bipolar disorder are treated with mood-stabilizing drugs such as lithium, carbamazepine, or valproic acid. ${ }^{10}$ One-third of patients treated with lithium show no recurrence of depressive or manic symptoms. ${ }^{10}$ Bipolar patients can also be treated with new mood-stabilizing drugs such as topiramate or lamotrigine or second-generation antipsychotic drugs such as quetiapine. ${ }^{11}$

\section{Overview of existing and emerging treatment options}

Second-generation antipsychotic drugs are commonly administered in the treatment of schizophrenia because they improve positive and negative schizophrenic symptoms. ${ }^{1}$ Typical neuroleptics or first-generation antipsychotic drugs such as haloperidol, a $\mathrm{D}_{2}$ antagonist, often cause extrapyramidal symptoms, and although they treat positive schizophrenic symptoms adequately their effects on negative schizophrenic and cognitive symptoms are more reduced. Accordingly, second-generation antipsychotic drugs, which are mostly $\mathrm{D}_{2}$ and $5-\mathrm{HT}_{2 \mathrm{~A}}$ antagonists, are preferred. While risperidone, which has greater affinity for the $\mathrm{D}_{2}$ receptor, still often causes extrapyramidal symptoms, olanzapine has a better effect on negative schizophrenic symptoms than other second-generation antipsychotic drugs. Quetiapine, which shows greater affinity for the $5-\mathrm{HT}_{2 \mathrm{~A}}$ receptor, has clinical effects comparable to those of other second-generation antipsychotic drugs. Currently, the injectable administration of aripiprazole, a partial $\mathrm{D}_{2}$ agonist, 5- $\mathrm{HT}_{2 \mathrm{~A}}$ antagonist and 5- $\mathrm{HT}_{1 \mathrm{~A}}$ agonist, is recommended for the long-term treatment of schizophrenic patients because it is well tolerated and safe for the treatment of positive, negative, and cognitive schizophrenic symptoms. ${ }^{12}$ The question that arises is whether a partial agonism at dopaminergic receptors, for example, the $\mathrm{D}_{2}$ receptor, might enable better tolerability and a better quality of life, since it does not interfere with dopaminergic receptors of the extrapyramidal system very much.

The treatment of bipolar disorder, that is, psychosis with depressive and manic symptoms, consists of the administration of mood-stabilizing drugs. Among first-generation drugs, lithium is the most effective one because one-third of patients show full remission of their depressive and manic symptoms. ${ }^{10}$ Other first-generation mood-stabilizing drugs are carbamazepine and valproate, which also have a prophylactic effect. Among responders to lithium, risk genes have been examined. These genes are associated with the neurotransmitters dopamine, serotonin, and glutamate; with second messengers such as cyclic adenosine-monophosphate; with protein kinase $\mathrm{C}$ pathways, and with substances involved in neuroprotection. ${ }^{9}$ Among the second-generation moodstabilizing drugs, the second-generation antipsychotic drugs risperidone and quetiapine, which block $\mathrm{D}_{2}$ and $5-\mathrm{HT}_{2 \mathrm{~A}}$ receptors in the mesolimbic system and hippocampus, should be mentioned. Lamotrigine is a drug of second choice or an add-on therapy in the treatment of bipolar disorder. In this disorder, it stabilizes hippocampal dopaminergic neurons and blocks NMDA and AMPA receptors. The function of dopaminergic and glutaminergic neurons has been described in the nigrostriatum and hippocampus..$^{10,13,14}$

\section{Review on the pharmacology, mode of action, and pharmacokinetics of cariprazine}

Cariprazine is a second-generation antipsychotic drug whose mechanism of action differs from that of second-generation antipsychotic drugs such as risperidone, olanzapine, and quetiapine. ${ }^{1}$ It exerts a partial agonism at $\mathrm{D}_{2}$ and $\mathrm{D}_{3}$ receptors, with a preferential binding for the $\mathrm{D}_{3}$ receptor. ${ }^{12,15}$ Moreover, cariprazine exerts a $5-\mathrm{HT}_{1 \mathrm{~A}}$ agonistic effect. Cariprazine has a tenfold greater affinity for the $\mathrm{D}_{3}$ receptor than for the $\mathrm{D}_{2}$ receptor and hence has different mechanisms of action than other second-generation antipsychotic drugs. Cariprazine exerts a partial agonism at dopaminergic receptors and does not block serotonergic subreceptors. In animal experiments, it shows antipsychotic and antimanic effects. It suppresses the spontaneous activity of mesolimbic dopaminergic neurons, 
but had no effect on nigrostriatal dopaminergic neurons. ${ }^{8}$ The partial agonism of cariprazine at $\mathrm{D}_{2}$ and at $\mathrm{D}_{3}$ receptors is achieved in the striatum and the prefrontal cortex. A sample of antipsychotic-naïve first-episode schizophrenia patients, who were treated with $\mathrm{D}_{2} / \mathrm{D}_{3}$ antagonist, showed normal sensory gating, that is, P50 gating and prepulse inhibition deficits. ${ }^{16}$ The data about pharmacokinetics of cariprazine can be summarized as follows. Cariprazine has a rapid absorption, and its mean half-time was $2-5$ days. ${ }^{8}$ Cariprazine has two major metabolites, namely desmethyl cariprazine and didesmethyl cariprazine, which have pharmacological properties similar to those of cariprazine. ${ }^{17}$ Cariprazine can be administered with or without food and its plasma peak concentration is reached in 3-5 hours. It is mainly eliminated via hepatic metabolism, like other lipophilic antipsychotics. Steady state is reached by cariprazine and desmethyl cariprazine after 1 week and after 4 weeks by didesmethyl cariprazine. ${ }^{17}$ After dose withdrawal, a 50\% reduction in plasma level is reached in about 1 week. No data are available in elderly patients or in patients suffering diseases of drug-eliminating organs. ${ }^{8}$ It is used for the treatment of schizophrenia and bipolar disorder. ${ }^{15}$ The most frequent adverse effects are as follows: insomnia, extrapyramidal disorder, akathisia, sedation, nausea, dizziness, and constipation. Mean changes in metabolic parameters are small and comparable to those observed in healthy volunteers. ${ }^{15}$

\section{Efficacy, safety, and tolerability issues}

Clinical trials have been carried out about the use of cariprazine in patients with schizophrenia and mania, in bipolar disorder. A randomized, clinical study of cariprazine and risperidone in a phase II study addressing the treatment of acute exacerbation of schizophrenia has been reported. ${ }^{15,18}$ A total of 1,011 patients were enrolled in the study, and 732 patients were randomized to receive treatment. The Positive and Negative Syndrome Scale (PANSS) total score changed from baseline to week 6 in patients receiving cariprazine, that is, the improvement of positive and negative schizophrenic symptoms was greater than that of placebo. ${ }^{15}$ Cariprazine at a dosage of 3 or $4.5 \mathrm{mg}$ /day was more efficient at improving the PANSS total score in comparison with the control group. The group receiving $1.5 \mathrm{mg}$ /day showed improvements from week 2 to week $6 .{ }^{12}$ Risperidone also afforded improvements in the PANSS total score as compared to the control group. The mean duration of treatment with the placebo was 30 days, 33 days for cariprazine, and 35 days for risperidone. ${ }^{15}$ Adverse effects occurred in $70 \%$ of the patients, in which insomnia, extrapyramidal disorder, akathisia, sedation, nausea, dizziness, and constipation were observed.
Serious side effects occurred in 19 patients, but there were no deaths. Weight gain was observed in a few patients receiving cariprazine, although it was more frequent in the group treated with risperidone. Patients receiving cariprazine had a slightly higher heart rate, and patients receiving risperidone had an elongation of the QT segment. Extrapyramidal symptoms such as parkinsonism and akathisia occurred more frequently in the group treated with risperidone. ${ }^{15}$

Haloperidol and second-generation antipsychotic drugs are also effective in the treatment of acute mania in bipolar disorder. Although these drugs have a safe therapeutic effect, adverse effects such as extrapyramidal symptoms, metabolic side effects, and weight gain are problems for the patients treated. The main question is whether cariprazine has a safe therapeutic effect for the treatment of acute mania in bipolar disorder. ${ }^{19}$ Altinbas et al performed a double-blind, randomized study with cariprazine, using doses from 3 to $12 \mathrm{mg} /$ day, in 238 patients with acute mania in bipolar disorder. ${ }^{19}$ Cariprazine improved the manic symptoms in week 3 in comparison with the control group receiving placebo. The most frequent adverse effects were extrapyramidal symptoms, headache, akathisia, constipation, nausea, and dyspepsia, which can be explained in terms of the partial dopamine agonism. Adverse effects observed in the treatment with other second-generation antipsychotic drugs, such as weight gain, metabolic disturbances, increases in prolactin levels, or QT prolongation, were not reported. The study revealed that manic symptoms were very much improved in comparison with the patients receiving placebo. ${ }^{19}$ However, adverse treatment-emergent acute effects such as akathisia and tremor can occur. Cariprazine has a greater antagonistic effect on the $\mathrm{D}_{2}$ receptor than aripiprazole and improves cognitive functions. Cariprazine has a therapeutic effect in acute mania in bipolar disorder like other second-generation antipsychotic drugs. ${ }^{19}$

\section{Patient-focused perspectives such as quality of life, patient satisfaction/ acceptability}

First, the effect of cariprazine on patients' quality of life during the treatment of an acute exacerbation of schizophrenia is described. ${ }^{8,15}$ Cariprazine, a new second-generation antipsychotic drug with a different pharmacological profile, improves all significant parameters, that is, the positive PANSS total score and the negative PANSS total score in comparison to placebo. ${ }^{15}$ The doses in the treatment of acute schizophrenia are $1.5-4.5 \mathrm{mg} /$ day (low dose) and 6-12.0 mg/day (high dose). ${ }^{20}$ Higher doses of cariprazine had a greater effect; they improved the total PANSS score better, and safety and 
tolerability were maintained. ${ }^{15}$ The therapeutic effect was comparable to that of risperidone, although the PANSS changes from baseline were greater on many parameters in the risperidone group. Patients receiving cariprazine underwent a smaller weight gain than patients receiving risperidone, and patients treated with cariprazine had no increases in prolactin levels, in contrast to the risperidone group. While second-generation antipsychotic drugs such as risperidone increase the risk of coronary artery heart disease, cariprazine does not increase cholesterol, triglycerides, or glucose levels. ${ }^{1,21}$ Cariprazine and risperidone caused extrapyramidal symptoms, and these occurred in a higher percentage in the risperidone group. ${ }^{15}$ Cariprazine has a greater affinity for the $\mathrm{D}_{2}$ and $\mathrm{D}_{3}$ receptors than aripiprazole and has a stronger antagonistic effect on the $\mathrm{D}_{2}$ receptor. ${ }^{22}$ Clinical trials should explore whether cariprazine exerts therapeutic effects comparable to those of aripiprazole. ${ }^{22}$

Second, the effect of cariprazine on patients' quality of life during the treatment of acute mania in bipolar disorder has been assessed. ${ }^{19}$ Cariprazine shows a good therapeutic effect and good safety and tolerability in the treatment of acute mania. Although second-generation antipsychotic drugs such as risperidone, olanzapine, and quetiapine elicit an increase in metabolic parameters, such as glucose, cholesterol, and triglycerides, and increase the risk of diabetes mellitus and coronary artery heart disease, cariprazine has a favorable metabolic profile because it binds only weakly to $H_{1}$ and $5-\mathrm{HT}_{2 \mathrm{C}}$ receptors. ${ }^{20}$ While risperidone is a prolactin-elevating antipsychotic drug, cariprazine is a prolactin-sparing drug. ${ }^{23,24}$ Cariprazine improves cognitive functions as a consequence of its high affinity for the $\mathrm{D}_{3}$ receptor. ${ }^{22}$ This is an important aspect in comparison with other second-generation antipsychotic drugs. Although cariprazine causes extrapyramidal symptoms less often than risperidone, akathisia occurs more often in the treatment with cariprazine than in placebo groups. ${ }^{25}$ All these results point to the good tolerability of cariprazine, although future clinical trials should be carried out in order to determine whether cariprazine improves patients' quality of life to a greater extent than other secondgeneration antipsychotic drugs.

\section{Conclusion, place in therapy}

Cariprazine is a recently developed second-generation antipsychotic drug with partial agonism at the $\mathrm{D}_{2}$ and $\mathrm{D}_{3}$ receptors: it has a tenfold greater affinity for the $\mathrm{D}_{3}$ receptor than for the $\mathrm{D}_{2}$ receptor, and it is recommended for the treatment of schizophrenia and acute mania in bipolar disorder. In the treatment of an acute exacerbation of schizophrenia, cariprazine treats positive and negative schizophrenic symptoms better than placebo and exerts an effect comparable to that of risperidone. Through partial agonism at the $\mathrm{D}_{3}$ receptor it improves cognitive functions. It causes extrapyramidal symptoms less often than risperidone, although acute akathisia and tremor can occur. In comparison with other second-generation antipsychotic drugs, it does not elicit changes in metabolic parameters that, if they did occur, could increase the risk for coronary artery heart disease or diabetes mellitus. Moreover, it does not raise prolactin levels, in contrast to risperidone. Low doses (1.5-4.5 mg/day) have the same therapeutic effect, safety, and tolerability as high doses (6-12 mg/day). However, one issue remains to be solved, that is, whether cariprazine has a safe therapeutic effect in the long-term treatment of schizophrenic patients.

In the treatment of acute mania in bipolar disorder, cariprazine improves the manic symptoms after 3 weeks as compared to the control group. The common adverse effects were extrapyramidal symptoms (acute akathisia and tremor can occur), constipation, nausea, and dyspepsia. Cariprazine shows a favorable metabolic profile. It elicits no weight gain in comparison with the control group and is a prolactinsparing antipsychotic drug. Two important points should be noted here. First, cariprazine is a promising antipsychotic drug for the treatment of schizophrenia, acute mania in bipolar disorder, and of schizophrenia with mania. In this context, clinical trials for the treatment of schizophrenic patients with manic symptoms remain to be carried out. The tolerability and the profile of adverse effects are remarkable in comparison to other second-generation antipsychotic drugs such as risperidone, olanzapine, and quetiapine because cariprazine shows a favorable metabolic profile and it does not raise prolactin levels. It causes extrapyramidal symptoms less often than other second-generation antipsychotic drugs such as risperidone. Second, it is important to know whether cariprazine maintains its good therapeutic effect and tolerability during the long-term treatment of schizophrenic, manic, and schizophrenic patients with mania.

Cariprazine, a new antipsychotic drug with a partial agonism at $\mathrm{D}_{2}$ and $\mathrm{D}_{3}$ receptors and $\mathrm{a}-\mathrm{HT}_{1 \mathrm{~A}}$ agonism was examined in clinical trials for the treatment of schizophrenia and mania in bipolar disorder. ${ }^{8,15,19}$ In clinical trials, cariprazine shows superiority to placebo on improving manic and positive and negative schizophrenic symptoms. This drug has a favorable metabolic profile, is a prolactin-sparing drug and does not cause electrocardiogram (ECG) changes, however, it often causes akathisia. ${ }^{15,19}$ In comparison with risperidone, it shows similar antipsychotic properties. ${ }^{15}$ However, 
the changes of psychotic symptoms from baseline, after administration of risperidone, were higher than those after administration of cariprazine. Clinical trials should be carried out to compare the effectiveness and safety of cariprazine with other second-generation antipsychotic drugs, above all aripiprazole. Moreover, long-term clinical trials should be undertaken in order to investigate the safety of cariprazine in the treatment of schizophrenia and bipolar disorder. ${ }^{8}$

\section{Acknowledgment}

The authors wish to thank Mr Nicholas Skinner (University of Salamanca, Spain) for supervising the English text.

\section{Disclosure}

The authors report no conflicts of interest in this work.

\section{References}

1. Werner FM, Coveñas R. Safety of antipsychotic drugs: focus on therapeutic and adverse effects. Exp Opin Drug Saf. 2014;13(8): 1031-1042.

2. Klosterkötter J. Indicated prevention of schizophrenia. Dtsch Arztebl Int. 2008;105(30):532-539.

3. Werner F-M, Coveñas R. Classical neurotransmitters and neuropeptides involved in schizophrenia: how to choose the appropriate antipsychotic drug? Curr Drug Ther. 2013;8(2):132-143.

4. Vasilyeva I, Biscontri RG, Enns MW, Metge CJ, Alessi-Severini S. Movement disorders in elderly users of risperidone and first generation antipsychotic agents: a Canadian population-based study. PLoS One. 2013;8(5):e64217.

5. McEvoy JP. Efficacy of risperidone on positive features of schizophrenia. J Clin Psychiatry. 1994;55 Suppl:18-21.

6. Maeda K, Lerdrup L, Sugino H, et al. Brexpiprazole II: antipsychoticlike and precognitive effects of a novel serotonin-dopamine activity modulator. J Pharmacol Exp Ther. 2014;250(3):605-614.

7. Korte SM, Prins J, Krajnc AM, et al. The many different faces of major depression: it is time for personalized medicine. Eur J Pharmacol. 2015;753:88-104.

8. Caccia S, Invernizzi RW, Nobili A, Pasina L. A new generation of antipsychotics: pharmacology and clinical utility of cariprazine in schizophrenia. Ther Clin Risk Manag. 2013;9:319-328.

9. Werner FM, Coveñas R. Classical neurotransmitters and neuropeptides involved in major depression: a review. Int J Neurosci. 2010;120(7): 455-470.

10. Rybakowski JK. Genetic influences on response to mood stabilizers in bipolar disorder: current status of knowledge. CNS Drugs. 2013; 27(3): 165-173.

11. Mula M, Cavanna AE, Monaco F. Psychopharmacology of topiramate: from epilepsy to bipolar disorder. Neuropsychiatr Dis Treat. 2006; 2(4):475-488.

Therapeutics and Clinical Risk Management

\section{Publish your work in this journal}

Therapeutics and Clinical Risk Management is an international, peerreviewed journal of clinical therapeutics and risk management, focusing on concise rapid reporting of clinical studies in all therapeutic areas, outcomes, safety, and programs for the effective, safe, and sustained use of medicines. This journal is indexed on PubMed Central, CAS,
12. Pilla Reddy V, Kozielska M, Suleiman AA, et al. Pharmacokineticpharmacodynamic modeling of antipsychotic drugs in patients with schizophrenia. Part I: the use of PANSS total score and clinical utility. Schizophr Res. 2013;146(1-3):144-152.

13. Rihmen Z, Gonda X, Kálmán J. Treatment of bipolar disorder with lamotrigine relapse and suicidal behaviour during 6 month follow-up. Neuropsychopharmacol Hung. 2015;17(1):7-13.

14. Calabresi P, Picconi B, Tozzi A, Ghiglieri V. Interaction between basal ganglia and limbic circuits in learning and memory processes. Parkinsonism Relat Disord. Epub 2015 Sep 5.

15. Durgam S, Starace A, Migliore R, Ruth A, Németh G, Laszlovszky I. An evaluation of the safety and efficacy of cariprazine in patients with acute exacerbation of schizophrenia: a phase II, randomized, clinical trial. Schizophr Res. 2014;152(2-3):450-457.

16. Düring S, Glenthoj BY, Andersen GS, Oranje B. Effects of dopamine D2/D3 blockade on human sensory and sensorimotor gating in initially antipsychotic-naive, first-episode schizophrenia patients. Neuropsychopharmacology. 2014;39(13):3000-3008.

17. Citrome L. Cariprazine: chemistry, pharmacodynamics, pharmacokinetics, and metabolism, clinical efficacy, safety, and tolerability. Expert Opin Drug Metabol Toxicol. 2013;9(2):193-206.

18. Veselinovic T, Paulzen M, Grunder G. Cariprazine, a new, orally active dopamine $\mathrm{D} 2 / 3$ receptor partial agonist for the treatment of schizophrenia, bipolar mania and depression. Expert Rev Neurother. 2013; 13(11):1141-1159.

19. Altinbas K, Guloksuz S, Oral ET. Clinical potential of cariprazine in the treatment of acute mania. Psychiatr Danub. 2013;25(3):207-213.

20. Zukin SR, Kane J, Cutler AJ, et al. Efficacy and safety of cariprazine in acute exacerbation of schizophrenia: a phase III, international, randomized, double-blind, placebo-controlled trial. Neuropsychopharmacology. 2012;38(Suppl 1):S319.

21. Daumit GI, Goff DC, Meyer JM, et al. Antipsychotic effect on estimated 10 -year coronary heart disease risk in the CATIE schizophrenia study. Schizophr Res. 2008;105(1-3):175-187.

22. Gyertyán I, Kiss B, Saghy K, et al. Cariprazine (RGH-188), a potent $\mathrm{D}_{2} / \mathrm{D}_{3}$ dopamine receptor partial agonist, binds to dopamine $\mathrm{D} 3$ receptors in vivo and shows antipsychotic-like and procognitive effects in rodents. Neurochem Int. 2008;59(6):925-935.

23. Kolotkin RL, Corey-Lisle PK, Crosby RD, et al. Impact of obesity on health-related quality of life in schizophrenia and bipolar disorder. Obesity (Silver Spring). 2008;16(4):749-754.

24. Kiss B, Horváth A, Némethy Z, et al. Cariprazine, (RGH-188), a dopamine $\mathrm{D}(3)$ receptor-preferring, $\mathrm{D}(3) / \mathrm{D}(2)$ receptor antagonistpartial agonist antipsychotic candidate: in vitro and neurochemical profile. J Pharmacol Exp Ther. 2010;333(1):328-340.

25. Seneca N, Finnema SJ, Laszlovsky I, et al. Occupancy of D2 and D3 and serotonin 5-HT1A receptors by the novel antipsychotic drug candidate cariprazine (RGH-188), in monkey brain measured using positron emission tomography. Psychopharmacology (Berl). 2011; 218(3):579-587.

\footnotetext{
Submit your manuscript here: http://www.dovepress.com/therapeutics-and-clinical-risk-management-journal
} 